\title{
DISEÑO Y EFECTIVIDAD DE LA "TRAMPA VIEYRA", PARA LA CAPTURA DE TUZAS VIVAS
}

\section{BÁRBARA VARGAS MIRANDA ${ }^{1}$ Y GERARDO CEBALLOS GONZÁLEZ ${ }^{2}$}

${ }^{1}$ Departamento de Ciencias de la Salud, UAM-Iztapalapa, Apartado Postal 55-535, México D.F. 09340, México. ${ }^{2}$ Instituto de Ecología, Universidad Nacional Autónoma de México. Apartado Postal 70-275, México, D. F. 04510, México. correo electrónico: gceballo@miranda.ecologia.unam.mx

\begin{abstract}
We introduce a trap to capture alive gophers which is called "Vieyra Trap". In comparison to other designs, we found a success rate up to 75\% larger than previous devices. Some advantages include the specific design for an endemic specie located in Lagos de Moreno, Jalisco, Mexico (Cratogeomys zinseri), it can be easily installed, minor ecological risks, and low cost.
\end{abstract}

Palabras claves: Trampa, diseño, México, tuzas.

Key word: Trap, drawing, Mexico, pocket gophers.

Existen limitaciones metodológicas en la captura de tuzas vivas, especialmente de los géneros Geomys, Orthogeomys y Pappogeomys, destacando el tamaño de los especimenes y sus hábitos cavadores, ya que pasan la mayor parte del tiempo en galerías subterráneas (Ingles, 1949). Se han descrito varios diseños de trampas para capturar tuzas vivas (Baker y William, 1972; Connor y Risch, 2009; Howard, 1952; Sargeant, 1966; Scheffer, 1934), entre los principales inconvenientes que presentan los diseños previos, destacan que requieren de grandes espacios para ser colocadas, son muy voluminosas y existe una perturbación considerable del hábitat, además presentan diseños complejos y están fabricadas con materiales costosos. Asimismo, los diseños de estas trampas en el trabajo de campo han demostrado ser pocas efectivas y complicadas de utilizarse.

Con el propósito de contribuir al mejoramiento de los métodos de captura de estas especies, en este trabajo se describe un nuevo diseño de trampa y se presentan datos de campo de su efectividad.

La "Trampa Vieyra", es de forma cilíndrica, elaborada con un tubo de PVC industrial de $3 \mathrm{~mm}$ de grosor. Mide $10 \mathrm{~cm}$ de diámetro y $50 \mathrm{~cm}$ de largo. En uno de los extremos, cuenta con una puerta perforada de acero inoxidable de 8x8 cm, sujeta por un perno del mismo material; la puerta se detiene con un dispositivo, el cual opera en cuanto entra el animal a la trampa. Dicho dispositivo consiste de un ganchillo unido a una platina a través de 
un hilo de pescar que corre a lo largo de la trampa y que se acciona cuando el animal entra al tubo, cerrando la puerta. En el otro extremo, se encuentra una rejilla de alambre de acero inoxidable, su función es la de no permitir la huida de la tuza y que el animal perciba que la galería esta abierta, lo que provoca a que se dirija al lugar a recubrirlo y que este quede atrapado en la trampa (Figura 1).

Se capturó un total de 35 individuos, procedentes de cuatro localidades. Se colectaron dos especies de tuzas,
Cratogeomys merriami y C. zinseri. Esta última especie, es un geómido endémico de México, registrada solamente en el municipio de Lagos de Moreno, Jalisco. El rendimiento promedio de colecta, fue de $79 \%$. Las trampas se colocaron por un promedio de 12 horas.

Se considero el rendimiento de la trampa, como el número de trampas colocadas entre el número de individuos colectados para cada una de las localidades, así como el promedio total de todos los lugares muestreados. El mayor rendimiento

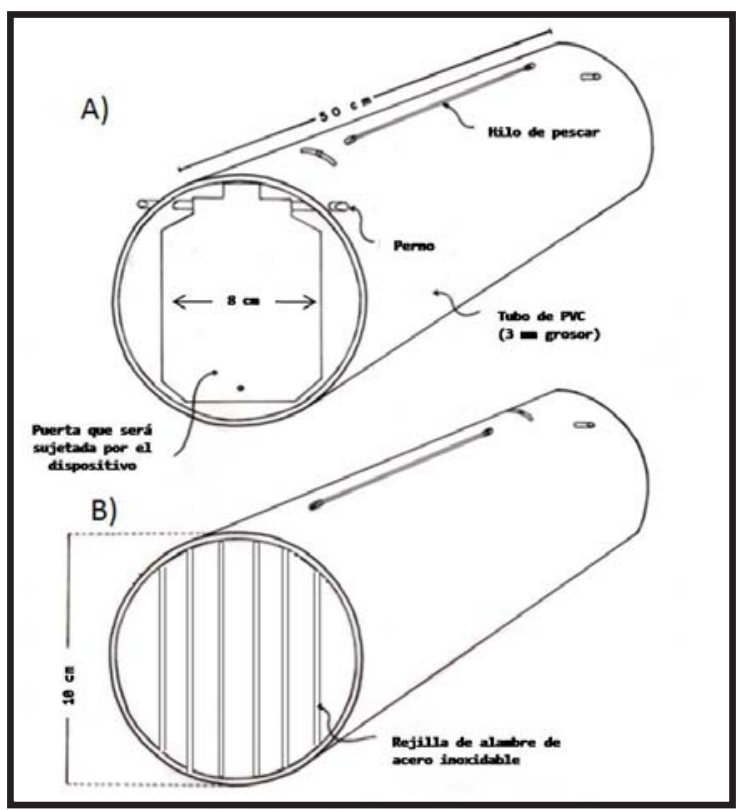

Figura 1. "Trampa Vieyra" para la captura de tuzas vivas. A) Vista frontal de la trampa, donde se muestra la puerta que se sujeta al dispositivo, B) Vista posterior de la trampa, donde se muestra la puerta fija. 
fue en la localidad de Hidalgo: Pachuca (90\%), de 10 trampas colocadas se capturaron nueve tuzas de la especie Cratogeomys merriami. En la localidad Parres, en el Distrito Federal, el rendimiento fue del 70\% (10 trampas colocadas y ocho individuos colectados de C. merriami). El rendimiento fue menor en la localidad Universidad Autónoma Metropolitana (UAMI): Distrito Federal, con un 60\%, de 20 trampas colocadas, se colectaron 15 individuos de la misma especie que en las dos últimas localidades. En Lagos de Moreno: Jalisco, se colectaron tres, $C$. zinseri, de cuatro trampas colocadas, teniendo el $75 \%$ de rendimiento.

Comparando los porcentajes de actividad del presente trabajo contra las cifras publicadas para los otros diseños. Connor y Risch (2009) mostro un éxito de captura del 25 al 50 \%; Baker y Williams (1972), tuvieron el $70 \%$ de rendimiento total. Nuestra trampa mostro una éxito de captura promedio de hasta un 75\% (60\% hasta $90 \%$ ), mayor al de los proyectos mencionados.

La familia Geomyidae es biológicamente única, por lo que es importante conocer más sobre su historia natural, tendencia evolutiva, sistemática, citogenética, fisiología y control como plaga, sin embargo, el primer factor limitante para su estudio es la dificultad para capturar ejemplares vivos (Ellison 1946), trabajos previos han descrito trampas para la captura de tuzas vivas (Baker y William, 1972; Connor y Risch, 2009; Howard, 1952; Sargeant, 1966; Scheffer, 1934), algunos de ellos presentan buen rendimiento de captura, pero sus diseños son muy elaborados. El reducido porcentaje de éxito para obtener resultados consistentes con varias especies de tuzas con las trampas ya descritas (especialmente del género Pappogeomys y Geomys), nos permitió sugerir un diseño nuevo de trampa.

La "Trampa Vieyra", demuestra con datos ser un diseño que permite tener mayor éxito y rendimiento de captura de las tuzas, además, su uso causa un menor deterioro ambiental debido a que su colocación no requiere de escavar grandes espacios (Baker y William, 1972; Connor y Risch, 2009; Howard, 1952; Sargeant, 1966; Scheffer, 1934), pues solo es necesario ubicar los túneles de las galerías e introducir la trampa. Además su costo de fabricación es económico, es de fácil colocación, está elaborada con material sencillo y es de fácil fabricación.

\section{AGRADECIMIENTOS}

Quisiéramos agradecer al Dr. Abraham Kobelkowsky Díaz profesor de la UAMI, por la elaboración de las figuras. Al Biól. Salvador Gaona Ramírez, a la M. en C. Carolina Müdespacher Ziehl y a Benjamín Vieyra por su apoyo y ayuda para las salidas al campo y probar la efectividad de la trampa.

\section{LITERATURACITADA}

Baker R.J. y S.L. William. 1972. A live trap for pocket gophers. Journal of Management, 36:1320-1322.

Connor, M.B. y T.S. Risch. 2009. Live Trap for Pocket Gophers. The Southwestern Naturalist, 54:100-103. 
Ellison, L.1946. The pocket gopher in relation to soil erosion on mountain range. Ecology, 27:101-114.

Howard, W.E. 1952. A live trap for pocket gophers. Journal of Mammalogy, 33:6165.

Ingles, L.G.1949. An improved live trap for pocket gophers. Murrelet, 30:55-56.

Sargeant, A.B.1966. A live trap for pocket gophers. Journal of Mammalogy, 47:729731.

Scheffer, T.H. 1934. Hints on live trapping. Journal of Mammalogy, 15:197-202. 